\title{
Transitioning self-regulated learning profiles in hypermedia-learning environments
}

\author{
Clarissa Lau ${ }^{1}$, Jeanne Sinclair ${ }^{2}$, Michelle Taub $^{3}$, Roger Azevedo $^{3}$, Eunice Eunhee Jang ${ }^{1}$ \\ ${ }^{1}$ Department of Applied Psychology and Human Development, Toronto, ON, Canada \\ ${ }^{2}$ Department of Curriculum, Teaching and Learning, University of Toronto, Toronto, ON, Canada \\ ${ }^{3}$ Department of Psychology, North Carolina State University, Raleigh, NC, USA
}

\{clarissa.lau, jeanne.sinclair, eun.jang\} @mail.utoronto.ca; $\{$ mtaub, razeved\} @ ncsu.edu

\begin{abstract}
Self-regulated learning (SRL) is a process that highly fluctuates as students actively deploy their metacognitive and cognitive processes during learning. In this paper, we apply an extension of latent profiling, latent transition analysis (LTA), which investigates the longitudinal development of students' SRL latent class memberships over time. We will briefly review the theoretical foundations of SRL and discuss the value of using LTA to investigate this multidimensional concept. This study is based on college students $(n=75)$ learning about the human circulatory system while using MetaTutor, an intelligent tutoring system that adaptively supports SRL and targets specific metacognitive SRL processes including judgment of learning (JOL) and content evaluation (CE). Preliminary results identify transitional probabilities of SRL profiles from four distinct events associated with the use of SRL.
\end{abstract}

\section{CCS Concepts}

- Applied computing Interactive learning environments

- Computing methodologies Latent variable models

\section{Keywords}

Self-regulated learning; Information-processing theory; Metacognition; Metamemory; Latent transition analysis

Permission to make digital or hard copies of all or part of this work for personal or classroom use is granted without fee provided that copies are not made or distributed for profit or commercial advantage and that copies bear this notice and the full citation on the first page. Copyrights for components of this work owned by others than ACM must be honored. Abstracting with credit is permitted. To copy otherwise, or republish, to post on servers or to redistribute to lists, requires prior specific permission and/or a fee. Request permissions from Permissions@acm.org.LAK '17, March 13-17, 2017, Vancouver, BC, Canada (C) 2017 ACM. ISBN 978-1-4503-48706/17/03 ...\$15.00

DOI: http://dx.doi.org/10.1145/3027385.3027443

\section{INTRODUCTION}

In dynamic learning environments such as intelligent tutoring systems and other advanced learning technologies, students continuously adapt to internal (e.g., prior knowledge) and external (e.g., effectiveness of systems; feedback and scaffolding) conditions by rapidly changing as they interact with the tasks and the system, based on accurate use of cognitive and metacognitive processes.

This progressive change occurs over multiple stages that require high-degree of regulation; however, previous research has indicated that students do not automatically develop an ability to regulate their SRL knowledge and skills [1,3]. This warrants a need to clarify how students initially develop regulatory behaviors and how they shift towards more advanced levels of regulation. This paper will investigate the transitional profiles of two aspects of self-regulated learning in the context of an online learning environment, MetaTutor.

\subsection{Self-regulated learning}

Over the course of engaging with learning in a technologically rich environment, students are engaged in high complex learning processes. Students are expected to acquire knowledge, respond to feedback, and evaluate progress - processes that require highlevels of regulation. In order to achieve success during the complex learning process, students must gain agency in directing their own learning. Self-regulated learning (SRL) describes students' accurate monitoring and regulation of effective cognitive, metacognitive, and motivational knowledge, skills, and strategies to learn, problem solve, and perform across tasks [12, 19]. SRL is a multi-phase process with the following assumptions: (1) students plan, monitor, control, and reflect their learning strategies and progress [12], and (2) this involves a three-stage cyclical process where students engage in orientation and planning phase, monitoring and performance phase, and evaluation and adaptation phase [18]. These theoretical models of SRL collectively suggest that a combination of knowledge, skills, and strategies are essential and occur in a sequential and/or cyclical manner during learning and problem solving. While these models do describe SRL as phases, only one model considers SRL as an event that unfolds over time - information-processing theory (IPT) $[16,17]$. Since this study is examining how SRL profiles transition over time, this study will draw specifically from the information-processing theory of SRL. 


\subsection{Information-processing theory of SRL}

According to the information-processing theory of SRL [16, 17], learning occurs through a series of four cyclical (yet not necessarily sequential) stages, in which information processing and SRL occur. In phase 1, task understanding, students must be able to clearly understand the task they are being asked to complete. In phase 2, goals and plans, students set appropriate goals for accomplishing the given task, along with plans for how they expect to achieve those goals. Phase 3, using learning strategies, involves students using the learning strategies (i.e., cognitive, metacognitive, and motivational SRL processes) they planned in the previous phase. In phase 4, adaptation, students can make changes to their goals, plans, and use of learning strategies to make sure they are using them efficiently. Furthermore, within each phase, information processing occurs as the interaction of one's conditions, operations, products, evaluations, and standards (COPES). Thus, according to this model, students control and monitor their use of information processing and SRL strategies during each phase of learning.

\subsection{Model of metamemory}

Nelson \& Narens' [10] model of metamemory suggests that metacognition occurs at a meta-level, which involves three phases of memory-related processes. Thus, engaging in these metacognitive processes at the meta-level can influence task performance at the object level. Specifically, at the encoding phase, students engage in ease of learning (EOL) judgments, where they anticipate how easy it will be to accomplish a task. At the retrieval phase, students are making judgments of their understanding of the material (i.e., judgment of learning or JOLs). At the retention phase, students make a retrospective confidence judgment (RCJ), where they judge their confidence in their performance on the task. According to this model, making these metacognitive judgments may impact students' task performance, via memory processes.

\subsection{Latent profiling of SRL}

A challenge in understanding SRL is capturing the interactive nature of information processing and SRL strategies that students deploy in real time during learning. Constructs such as SRL are often difficult to observe directly, so traditional research methods (e.g., self-reports) will typically infer this behavior after the task is completed. However, these methods can hardly capture the dynamic and progressive stages of SRL. Recent advances have led researchers to use on-line trace methods (e.g., log-files, concurrent think-alouds) to measure the real-time deployment of SRL processes during learning $[1,2]$. The primary difference between traditional techniques and latent profiling is: "the common factor model decomposes the covariances to highlight relationships among the variables, whereas the latent profile model decomposes the covariances to highlight relationships among students" [4, p. 6]. Latent profiling is a latent variable model that identifies unobserved subgroups in a population. One study established distinct latent profiles of SRL that exist across students and these profiles are associated with varying academic outcomes [3]. Findings from this study suggested that individuals with minimal and disorganized profiles of SRL might demonstrate poorer academic outcomes [3]. This approach is a person-centered analysis that is more appropriate for understanding a multi-phased concept such as SRL.
This study is situated to investigate the unique characteristics of students' self-regulatory learning behaviors at different stages of interaction in the online learning environment, MetaTutor. During MetaTutor, metacognitive judgments during learning are measured by assessing students' level of confidence in making these judgments. This study aims to elucidate unique student profiles of SRL and describe the changing states across time.

\section{METHOD}

\subsection{Participants}

A total of 194 students are included in the study with 99 in the control group and 95 in the experimental group. From the experimental group, a total of 75 students are included in this ongoing study. Students were recruited from three major universities across North America. Background information was available for only 70 students. Of the 70 students, 53\% were males and $47 \%$ were females. Mean age was $M=20.11(S D=$ $.21)$ and mean GPA was $M=3.31(S D=.09)$.

\subsection{MetaTutor}

This study is set in the context of MetaTutor, an intelligent tutoring system that is built upon theoretical models of SRL [1, 13, 16, 17, 18]. The system engages students in biological concepts (e.g., the human circulatory system) and provides support through offering various strategies to plan, monitor, and reflect upon their learning. Students are prompted to set goals with respect to their learning and engage in cognitive and metacognitive SRL strategies while the system provides feedback based on student scores on quizzes and responses to making metacognitive judgments, such as JOLs. During the process of reading and inspecting hypermedia materials, students are given an option to select cognitive learning strategies, such as summarizing (SUMM), taking notes (TN), planning (PLAN), prior knowledge activation (PKA), and making inferences (INF), and metacognitive monitoring processes such as monitoring progress towards goals (MPTG), judgment of learning (JOL), feeling of knowing (FOK), and content evaluation (CE) from an SRL palette provided by the system's interface. These processes include: This study will examine two of these processes: judgment of learning (JOL) and content evaluation (CE). Below is a screenshot of the MetaTutor main interface (See Figure 1). The interface includes elements designed to foster effective SRL, such as a timer (top left), a table of contents (left, below the timer), content (center), overall learning goal and sub-goals (top center), a pedagogical agent (top right), and the SRL palette (right, below the agent).

Participants' background knowledge of biological concepts ranged from no experience to some experience (i.e., taken 1-5 undergraduate science courses). Prior to learning, participants were randomly assigned to one of two experimental conditions: prompt and feedback, or control. In the prompt and feedback condition, participants were prompted to use cognitive and metacognitive SRL strategies, and were provided with immediate feedback on their performance. Additionally, participants could self-initiate the use of these SRL processes, and were still provided feedback on their performance. In the control condition, participants were not prompted to engage in SRL strategies, nor were they provided with feedback if they self-initiated the use of these processes. As such, participants in the control condition were left to work independently without being provided with any guidance from the system. 


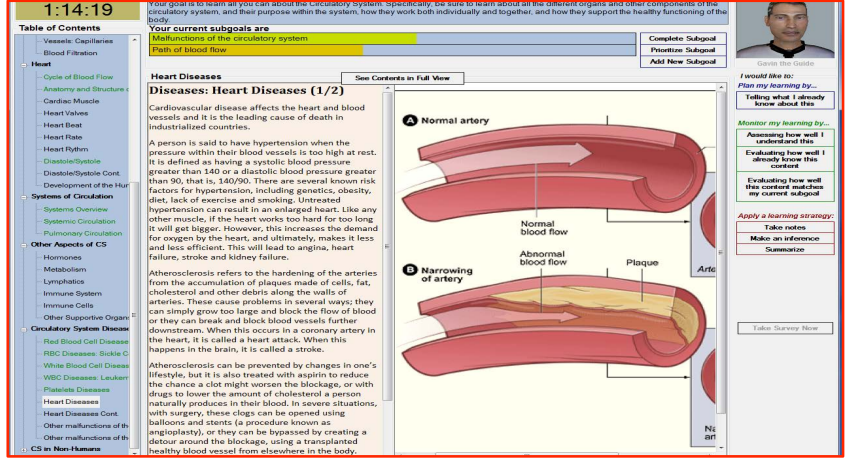

Figure 1. Main interface of MetaTutor tutoring system.

\subsection{Metacognitive monitoring process}

For this analysis, we selected the metacognitive monitoring processes: judgment of learning (JOL) and content evaluation (CE), as research has shown these processes to be influential in learning about complex science topics $[1,5,7]$.

\subsubsection{Judgment of learning}

Judgments of learning (JOLs) require students to assess their understanding of the material they are currently reading $[1,2,5$, 7]. This strategy requires learners to monitor their comprehension of the material, which is an effective strategy as it makes them actively aware of their progress during learning. Moreover, once this judgment is made, students can select the appropriate subsequent cognitive learning strategies to use, based on the valence of that judgment (i.e., JOL+: I understand this or JOL-: I do not understand this). For example, a student who does understand the material can go on to take notes on the content (i.e., cognitive strategy of taking notes) compared to a student who does not understand the material, who should re-read the material as the subsequent cognitive learning strategy. It is important to emphasize that valence is associated with the accuracy of a metacognitive judgment and we can therefore assume that a metacognitive-strategy dyad can either be adaptive (e.g., a JOL- followed by re-reading the same materials) or maladaptive (e.g., a JOL- followed by reading additional research).

\subsubsection{Content evaluation}

Content evaluations (CEs) involve students assessing whether the current content is relevant to the sub-goal they are working on [1, $2,6,7]$ making them aware of the relevancy of the material they are reading. When making a $\mathrm{CE}$, the student evaluates the relevancy of the text and the diagram on the content page, thereby monitoring the material they are reading to accomplish their subgoals. Similar to JOLs, there is also a valence component associated with making this metacognitive evaluation, such that the content can be relevant to the current sub-goal (i.e., $\mathrm{CE}+$ ), which should be followed by continuing to read that page, inspect the diagram, and take notes or summarize the material. In contrast, an evaluation of the content as being irrelevant to the current sub-goal (i.e., CE-), would be followed by changing pages to a relevant one.

Many research studies investigating the impact of JOLs on learning have revealed that making these judgments can positively impact learning $[1,5]$. One main focus of research on JOLs has been on assessing how students' levels of confidence (i.e., the judgments they make) impact their subsequent learning [5]. Additionally, research investigating the use of CEs has suggested that selecting more relevant content pages obtain higher performance scores [14] Although this research demonstrates the importance of judging one's understanding of content and selecting relevant pages for completing sub-goals, research is lacking on assessing how the accuracy of these judgments change over time.

\subsection{Latent transition analysis}

Latent transition analysis (LTA) is an extension of latent class analysis (LCA) that identifies underlying traits longitudinally. LCA is a statistical model that groups individuals together based on unobserved traits (i.e. latent traits) that are inferred with categorical variables [6]. LCA outputs latent classes that describe distinct behaviors shared by a group of individuals. Extending from LCA, LTA describes changes in these latent classes across time and transitional probabilities are estimated. These latent classes are not assumed to be static, but rather, individuals may be described with different classes at different times.

The most appropriate LTA model for describing a population of individuals sharing similar behaviors is estimated with three sets of parameters. First, latent status membership probabilities are estimated for subsequent times after the first time point. These probabilities recognize the proportion of individuals that would belong in any given latent class. Second, transition probabilities are calculated to identify the chance of students changing from one latent class from one time point to another latent class at the next time point. Third, model-fit indices are used to determine the relationship between the observed indicates and the latent class. Three model-fit indices are typically used: likelihood-ratio $G^{2}$ fit statistic, Akaike Information Criteria (AIC), and Bayesian Information Criteria (BIC). For interpretation, larger values of likelihood-ratio $G^{2}$ value indicate rejection of the null-hypothesis [9] while smaller values of AIC and BIC are indicators of better model fit [3]. For this study, LTA was conducted in SAS with the PROC LTA procedure [8].

\section{RESULTS}

Latent transition analysis for 2-, 3-, and 4- class solutions was conducted. The two-class model was considered due to low AIC and BIC values, but classes and transitions lacked interpretability. The selected model is the three-class solution, as it was the most parsimonious, had the best goodness of fit statistics of interpretable models, and offered the most useful interpretation. Table 1 indicates the model fit statistics for the 2-, 3-, and 4-class models.

According to this model, all members of Class $1(n=20)$ correctly identified content relevant to their sub-goal (CE). Approximately $58 \%$ of this class judged their learning (JOL) with correct confidence or under-confidence in light of their page quiz results, while $42 \%$ were overconfident. Thus this class has strength in CE and emerging skills in JOLs. The members of Class $2(n=39)$ have the contrasting profile: all of Class 2 exhibited accurate or underconfident JOL, but only approximately $55 \%$ of Class 2 correctly identified the relevance of page/image content for their sub-goal. As such, class 2 exhibits strength in JOL and emerging CE. Class 3 consists of learners who exhibit challenges in both JOLs and CEs. $100 \%$ of Class $3(n=16)$ learners exhibited overconfidence in JOL, and $100 \%$ also incorrectly identified content relevant to their 
current learning sub-goal (CE). Classes are constrained to have the same composition over the two time points.

Table 1. Comparison of LTA models.

\begin{tabular}{|c|c|c|c|c|}
\hline $\begin{array}{c}\text { Number } \\
\text { of classes }\end{array}$ & $\boldsymbol{G}^{\mathbf{2}}$ & $\mathbf{d f}$ & $\mathbf{A I C}$ & BIC \\
\hline 2 & 6.813 & 8.000 & 20.813 & 37.035 \\
\hline $\mathbf{3}$ & $\mathbf{1 . 8 3 3}$ & $\mathbf{1 . 0 0 0}$ & $\mathbf{2 9 . 8 3 3}$ & $\mathbf{6 2 . 2 7 8}$ \\
\hline 4 & 0.000 & -8.000 & 46.000 & 99.302 \\
\hline
\end{tabular}

Note: Bold font indicates the selected model.

Table 2. Item-response probabilities (probability of item response given latent status), prevalence of latent statuses, and transition probabilities in latent status membership.

\begin{tabular}{|c|c|c|c|}
\hline & \multicolumn{3}{|c|}{ Latent Status } \\
\hline & $\begin{array}{l}\text { Class 1 } \\
(n=20) \text { : } \\
\text { Strong } \\
\text { CE, } \\
\text { emerging } \\
\text { JOL }\end{array}$ & $\begin{array}{l}\text { Class } 2 \\
(n=39) \text { : } \\
\text { Strong } \\
\text { JOL, } \\
\text { emerging } \\
\text { CE }\end{array}$ & $\begin{array}{l}\text { Class } 3 \\
(n=16) \text { : } \\
\text { Overconfident } \\
\text { JOL, } \\
\text { incorrect } \\
\text { CE }\end{array}$ \\
\hline \multicolumn{4}{|l|}{$\begin{array}{l}\text { Item-response } \\
\text { probabilities: }\end{array}$} \\
\hline \multicolumn{4}{|l|}{ JOL } \\
\hline $\begin{array}{l}\text { Under or correct } \\
\text { confidence }\end{array}$ & 0.576 & $>0.999$ & $<0.001$ \\
\hline Overconfidence & 0.424 & $<0.001$ & $>0.999$ \\
\hline \multicolumn{4}{|l|}{$\mathrm{CE}$} \\
\hline Incorrect & $<0.001$ & 0.455 & $>0.999$ \\
\hline Correct & 0.999 & 0.545 & $<0.001$ \\
\hline \multicolumn{4}{|l|}{$\begin{array}{l}\text { Prevalence of statuses } \\
\text { at: }\end{array}$} \\
\hline Time 1 & 0.269 & 0.517 & 0.213 \\
\hline Time 2 & 0.454 & 0.478 & 0.066 \\
\hline \multicolumn{4}{|l|}{$\begin{array}{l}\text { Transitions from Time } \\
1 \text { (rows) to } \\
\text { Time } 2 \text { (columns): }\end{array}$} \\
\hline $\begin{array}{l}\text { Class 1: Strong } \\
\text { CE, emerging JOL }\end{array}$ & 0.370 & 0.630 & $<0.001$ \\
\hline $\begin{array}{l}\text { Class 2: Strong } \\
\text { JOL, emerging CE }\end{array}$ & 0.617 & 0.280 & 0.103 \\
\hline $\begin{array}{l}\text { Class 3: } \\
\text { Overconfident JOL } \\
\text { and } \\
\text { incorrect CE }\end{array}$ & 0.168 & 0.769 & 0.062 \\
\hline
\end{tabular}

Note: Item-response probabilities are constrained to be equal at time points 1 and 2. Bold font indicates membership in the same latent status at both time points.

According to Table 2, there were more than $75 \%$ of students in Class 3 who demonstrated difficulty with both JOL and CE. Of this group, more than $75 \%$ improved their judgment of learning, and approximately $17 \%$ improved their content evaluation skills. Just $6 \%$ of the original Class 3 remained in Class 3. Overall, the probability of being in this class decreased approximately $15 \%$ from the initial measurement in time point 1 to the final measurement in time point 2 . This is a remarkable finding, considering their time with the MetaTutor program was limited to only a few hours at most.

Table 2 also indicates that approximately $63 \%$ of students in Class 1 (Strong CE/Emerging JOL) transitioned to Class 2 (Strong $\mathrm{JOL} /$ Emerging $\mathrm{CE}$ ) at time point 2 , while $61 \%$ of students did the reverse. There was less than 0.001 probability that students in Class 1 transitioned to Class 3 (Overconfident JOL/Incorrect CE), but approximately $10 \%$ of Class 2 did transition to Class 3 , indicating that they were more likely than Class 1 to exhibiting overconfidence and incorrect $\mathrm{CE}$ after engaging with the MetaTutor system.

\section{DISCUSSION}

This ongoing research is intended to elucidate the transitioning profiles of SRL in the context of MetaTutor. Extending from previous literature investigating the construct and latent nature of SRL $[3,11,12]$, this study investigates the changing latent SRL profiles over two time points.

Preliminary results indicated that a three-class solution best fit the model. It is important to remember that transitioning from Class 1 (strong $\mathrm{CE}$, emerging JOL) to 2 (strong JOL, emerging $\mathrm{CE}$ ), or the reverse, does not indicate that there has been a weakening of the other skill. Students transitioning from one class to another class are now demonstrating a different profile of JOLs and CEs. The findings of this study indicate that students exhibit different combinations of SRL behaviors across short time periods. Further inferential statistics are required to clarify which combinations maximize performance outcomes.

Class 3 (i.e., learners who demonstrate challenges in both JOL and $\mathrm{CE}$ ) is an interesting profile to mention. More than $75 \%$ of this class later demonstrated the significant improvements in their JOL and approximately $17 \%$ of these students improved their CE. An explanation may be found in the tailored feedback provided by MetaTutor. The feedback given by MetaTutor is tailored specifically to students' input, with over 20 different feedback responses. The time that students spend engaging in MetaTutor improves their outcomes relative to their area(s) of weakness. For example, of those who were in Class 1 at time point 1 (emerging JOL), more than half made gains in JOL, ending up in Class 2. A similar result is exhibited for Class 2, with over half of the members of Class 2 at time point 1 transitioning to Class 1 at time point 2 . This can potentially be attributed to the tailored feedback that students received in their area of weakness as they engaged with MetaTutor. In support of this thought, an earlier study by Thompson [15] found that general feedback did not significantly improve accuracy of students' confidence-recall, but instead found that students' confidence-recall improved when feedback was given for specific types of questions.

We recognize a limitation to this preliminary study. JOL and CE events were only included when feedback was provided. Therefore, students who were in the control condition were not included in the preliminary analysis and the sample size was reduced.

\subsection{Next Steps}

We do intend to continue the analysis by investigating predictive relationships between the transitioning SRL profiles and performance outcomes (e.g., setting sub-goals, time spent on task, 
and outcome on quiz and post-test scores). Next, the current constructs of JOL and CE have been dichotomized for the preliminary analysis; however, JOL and CE constructs do consist of more than one dimension (e.g., relevancy of text vs. relevancy of diagram). Further investigation would be required to appropriate categorize these constructs so these constructs can be more accurately represented in the analysis. Finally, the nature of feedback is particularly interesting and would require further investigation to understand how different students demonstrating various SRL profiles would respond to tailored feedback.

\section{ACKNOWLEDGMENTS}

The research presented in this paper has been supported by funding from National Science Foundation (DRL1431552) and the Social Sciences and Humanities Research Council of Canada (SSHRC 895-2011-1006). Any opinions, findings, and conclusions or recommendations expressed in this material are those of the author(s) and do not necessarily reflect the views of the National Science Foundation or Social Sciences and Humanities Research Council of Canada. The authors of this study would like to acknowledge Nicholas Mudrick for his involvement with MetaTutor.

\section{REFERENCES}

[1] Azevedo, R., Harley, J., Trevors, G., Duffy, M., FeyziBehnagh, R., Bouchet, F., and Landis, R. S. 2013. Using trace data to examine the complex roles of cognitive, metacognitive, and emotional self-regulatory processes during learning with multi-agent systems. In R. Azevedo \& $\mathrm{V}$. Aleven, Eds. International handbook of metacognition and learning technologies, Amsterdam, The Netherlands: Springer 2013, 427-449.

[2] Azevedo, R., Moos, D. C., Johnson, A. M., and Chauncey, A. D. 2010. Measuring cognitive and metacognitive regulatory processes during hypermedia learning: Issues and challenges. Educational Psychologist, 45, 4, 210-223.

[3] Barnard-Brak, L., Lan., W. Y., and Paton, V. O. 2010. Profiles in self-regulated learning in the online learning environment. The International Review of Research in Open and Distributed Learning, 11, 1, 61-80.

[4] Bauer, D. J., and Curran, P. J. 2004. The integration of continuous and discrete latent variable models: Potential problems and promising opportunities. Psychological Methods, 8, 338-363.

[5] Dunlosky, J., and Lipko, A. 2007. Metacomprehension: A brief history and how to improve its accuracy. Current Directions in Psychological Science, 16, 228-232.

[6] Goodman, L. A. 1974. Exploratory latent structure analysis using both identifiable and unidentifiable models. Biometrika., 61, 215-231.

[7] Greene, J. A., and Azevedo, R. 2009. macro-level analysis of SRL processes and their relations to the acquisition of sophisticated mental models. Contemporary Educational Psychology, 34, 18-29.

[8] Lanza, S. T., and Collins, L. M. 2010. A new SAS procedure for latent transition analysis: Transitions in dating and sexual risk behavior. Dev Psychol, 44, 2, 446-456.

[9] Lanza, S. T., Dziak, J. J., Huang, L., Xu, S., and Collins, L. M. 2011. Proc LCA \& Proc LTA users 'guide (Version 1.2.6). University Park, PA: The Methodology Center, Penn State University. Retrieved from http://methodology.psu.edu

[10] Nelson, T. O., and Narens, L. 1990. Metamemory: A theoretical framework and new findings. The Psychology of Learning and Motivation, 26, 125-173.

[11] Pintrich, P. R. 2000. The role of goal orientation in selfregulated learning. In M. Boekaerts, P. Pintrich, \& M. Zeidner, Eds. Handbook of self-regulation, New York, NY: Academic Press, 452-502.

[12] Pintrich, P. R., and De Groot, E. V. 1990. Motivational and self-regulated learning components of classroom academic performance. Journal of Educational Psychology, 82, 1, 3340.

[13] Schunk, D. 2005. Self-regulated learning: The educational legacy of Paul R. Pintrich. Educational Psychologist, 40, 8594.

[14] Taub, M., Azevedo, R., Bouchet, F., Clodfelter, E., and Mudrick, N. 2014. Can scaffolds from pedagogical agents influence effective completion of sub-goals during learning with a multi-agent hypermedia-learning environment? In Proceedings of the 11th International Conference of the Learning Sciences (Boulder, Colorado 2014), 1052-1056.

[15] Thompson, W. B. 1998. Metamemory accuracy: Effects of feedback and the stability of individual differences. American Journal of Psychology, 111, 1, 33-42.

[16] Winne, P., and Hadwin, A. 1998. Studying as self-regulated learning. In D. Hacker, J. Dunlosky, \& A. Graesser, Eds. Metacognition in educational theory and practice, Mahwah, NJ: Erlbaum, 227-304.

[17] Winne, P., and Hadwin, A. 2008. The weave of motivation and self-regulated learning. In D. Schunk \& B. Zimmerman, Eds. Motivation and self-regulated learning: Theory, research, and applications, Mahwah, NJ: Erlbaum, 297-314.

[18] Zimmerman, B. J. 1998. Developing self-fulfilling cycles of academic regulation: An analysis of exemplary instructional models. In D. H. Schunk, \& B. J. Zimmerman, Eds. Selfregulated learning: from teaching to self-reflective practice, New York: Guilford Press, 1-99.

[19] Zimmerman, B. J. 2008. Investigating self-regulation and motivation: Historical background, methodological developments, and future prospects, American Educational Research Journal, 45, 166-183. 